www.jmscr.igmpublication.org

Impact Factor (SJIF): 6.379

Index Copernicus Value: 71.58

ISSN (e)-2347-176x ISSN (p) 2455-0450

crossref DOI: https://dx.doi.org/10.18535/jmscr/v6i6.23

Journal Of Medical Science And Clinical Research

IGM Publication

An Official Publication of IGM Publication

\title{
Prospective Study of Predictive Factors and Outcome in Sperm Retrieval of Azoospermic Males: Initial Experience in Tertiary Care Centre
}

\author{
Authors \\ Dr Deepak Kumar ${ }^{1}$, Dr Namita Gupta ${ }^{2 *}$ \\ ${ }^{1}$ Classified Specialist (Surgery) \& Urologist, Command Hospital (Southern Command), Pune \\ ${ }^{2}$ Associate consultant (Obstetrics and Gynaecologist), Ruby Hall Clinic (Wanowarie), Pune \\ *Corresponding Author \\ Dr Namita Gupta
}

Associate consultant (Obstetrics and Gynaecologist), Ruby Hall Clinic (Wanowarie), Pune, India

\begin{abstract}
Parenthood is one of the most universally desired goals in adulthood. After varicocele, azoospermia is the second major cause of infertility in men. Sperm retrieval in combination with IVF/ICSI is the only medical procedure for an azoospermic man to father a child. Various techniques have been described for sperm retrieval through testicles in azoospermic males. They include open surgery methods like open testicular biopsy (open TESE), MESA (Microscopic epididymal sperm aspiration), micro-TESE (Microscopic Testicular sperm extraction) and percutaneous methods like TESE (Testicular sperm extraction), PESA (Percutaneous epididymal sperm aspiration), TEFNA (Testicular fine needle aspiration).

Methods: This study was designed as a hospital based descriptive prospective study with the aim to find clinical, biochemical and intraoperative predictive factors for successful sperm retrieval and their predictive values amongst successful sperm retrieval.

Results: In our study of 80 azoospermic males 9 (11.25\%) were diagnosed as obstructive azoospermia \& $71(88.75 \%)$ were diagnosed as non-obstructive azoospermia patients. Difference in BMI is not statistically significant between these groups.The mean FSH, LH, Testosterone and Prolactin in non-obstructive and obstructive azoospermia patients with positive sperm retrieval was significant. Out of 71 patients with nonobstructive azoospermia TESE was successful in retrieving sperms in 28 patients (39.4\%). Out of 43 unsuccessful TESE patients micro TESE was done in 34 patients and sperms could be found in 8 patients $(23.5 \%)$.

Conclusion: Age, BMI among successful and unsuccessful sperm retrieval groups are comparable and don't show any statistically significant difference. Whereas FSH, LH, Prolactin levels and mean testis volume show statistically significant difference between successful and unsuccessful sperm retrieval patients. Sperm retrieval for obstructive azoospermia gives excellent result with PESA. For non-obstructive azoospermia patients sperm retrieval can be achieved in around 50\% of cases. Micro TESE increases overall sperm retrieval rates by around $12 \%$ when done after failed TESE. Microscopic seminiferous tubule appearance can be used to predict chance of sperm retrieval but requires validation by further studies and clinical pregnancy rates of around 50\% can be achieved by using surgically retrieved sperms.

Keywords: obstructive and non obstructive azoospermia, Sperm retrieval technique, IVF-ICSI (Invitro fertilization- intracytoplasmic sperm injection).
\end{abstract}




\section{Introduction}

Parenthood is one of the most universally desired goals in adulthood. However, many couples will need medical help to resolve underlying fertility problems. WHO describes infertility as "a disease of the reproductive system defined by the failure to achieve a clinical pregnancy after 12 months or more of regular unprotected sexual intercourse."(1) Male infertility refers to a male's inability to cause pregnancy in a fertile female. In humans $20-30 \%$ of infertility cases are due to male infertility, 20$35 \%$ are due to female infertility, and $25-40 \%$ are due to combined problems in both partners. Thus male factor accounts for $40-50 \%$ of infertility. ${ }^{(2)}$ It affects approximately $7 \%$ of all men. ${ }^{(3)}$ According to WHO the prevalence of primary infertility among men in India is around 3\%.(4)

The main cause of male infertility is low semen quality either in the form of low count or low motility but a proportion of men will have no sperms in semen. After varicocele, azospermia is the second major cause of infertility in men. Azoospermia, defined as the absence of sperm in the ejaculate, is identified in approximately $1 \%$ of all men and in 10 to $15 \%$ of infertile males. ${ }^{(5)}$ Azoospermia may occur because of reproductive tract obstruction (obstructive azoospermia) or inadequate production of spermatozoa, such that spermatozoa do not appear in the ejaculate (nonobstructive azoospermia). ${ }^{(6)}$

Obstructive azoospermia can also be called as post-testicular azoospermia and is mainly caused due to physical obstruction of the posttesticular genital tracts. In it the sperms are produced but are not ejaculated. It affects around $30 \%(7 \%-51 \%)$ of azoospermic men. ${ }^{(7)}$ The most common reason is a vasectomy done to induce contraceptive sterility. Other obstructions can be congenital,such as agenesis of the vas deferens as seen in certain cases of cystic fibrosis or acquired, such as ejaculatory duct obstruction due to infection. ${ }^{(6,7)}$

Non-obstructive azoospermia is divided into pretesticular azospermia or testicular azospermia. Pretesticular azospermia is characterized by inadequate stimulation of otherwise normal testicles and genital tract. Follicle-stimulating hormone (FSH) levels are low (hypogonadotropic) leading to inadequate stimulation of the testes to produce sperm. Examples include hypopituitarism, hyperprolactinemia etc.

Pretesticular azoospermia is seen in about $2 \%$ of azoospermia. ${ }^{(7)}$ In testicular azospermia the testes are abnormal, atrophic, or absent, and sperm production is severely disturbed or absent. FSH levels tend to be elevated due to lack of feedback inhibition on FSH. The condition is seen in 49$93 \%$ of men with azoospermia. ${ }^{(7)}$ Testicular failure includes absence of production as well as low production and maturation arrest during the process of spermatogenesis. Causes for testicular failure include congenital issues such as in certain genetic conditions (e.g. Klinefelter syndrome), some cases of cryptorchidism or Sertoli cell-only syndrome as well as acquired conditions by infection (orchitis), surgery (trauma, cancer), radiation, or other causes. ${ }^{(6,7)}$

Sperm retrieval in combination with IVF/ICSI is the only medical procedure for an azoospermic man to father a child. Patients with acquired obstruction of the male reproductive tract may be treated using microsurgical reconstruction or transurethral resection of the ejaculatory ducts, depending on the level of obstruction.

Alternatively, sperm retrieval with assisted reproduction may be used to effect pregnancies, with success rates of $25-65 \%$ reported by different centres. ${ }^{(6)}$ Non-obstructive azoospermia may be treated by defining the cause of low sperm production and initiating treatment. In these men sperm retrieval from the testis may be effective in $30-70 \%$ of cases. Once sperm are found, pregnancy rates of $20-50 \%$ may be obtained at with in vitro fertilisation and intracytoplasmic sperm injection. ${ }^{(6,8)}$

Intracytoplasmic sperm injection (ICSI) is an artificial technique of reproduction where a single sperm can be physically injected into an egg. Because of this technique $90 \%$ of all infertile men have the potential to conceive their own genetic child. ${ }^{(8)}$ ICSI is generally performed as a part of in 
vitro fertilization procedure after extracting one to several oocytes from a woman. The procedure is done under a microscope using multiple micromanipulation devices. The sperm is released directly into the oocyte using micropipette. ${ }^{(9)}$ After

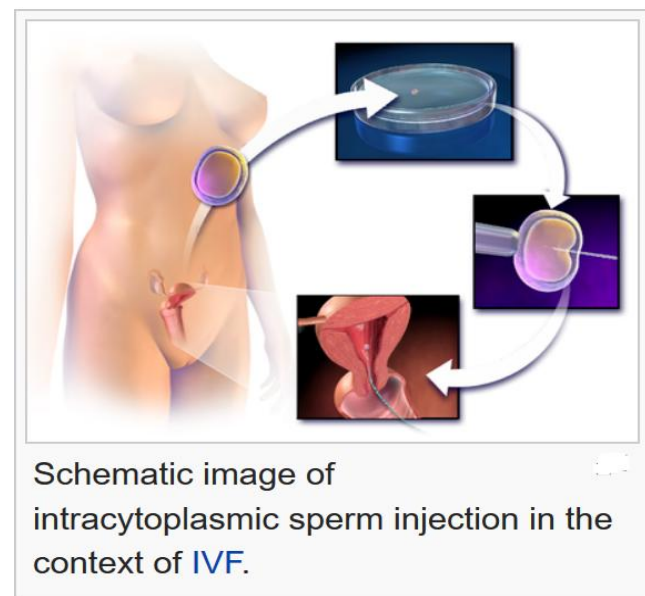

the procedure, the oocyte is placed into cell culture and checked for signs of fertilization. Once fertilized, the embryo is implanted into female uterus for development.

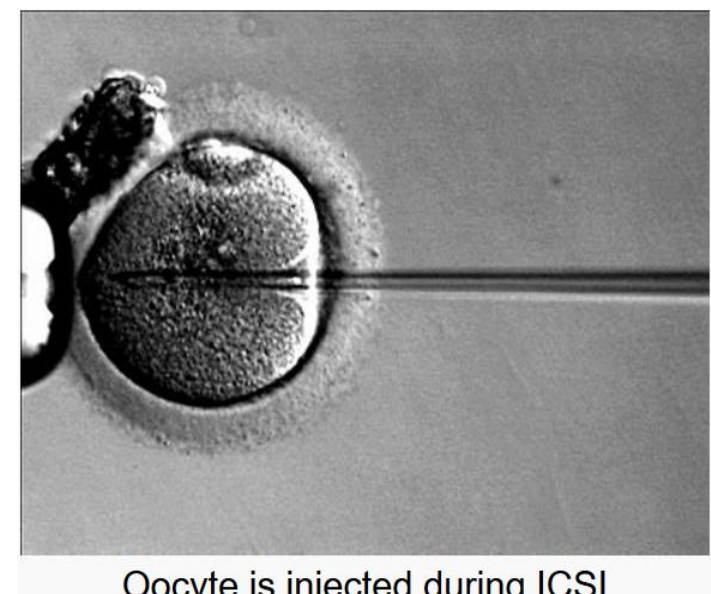

Oocyte is injected during ICSI

Fig 1 IVF - ICSI

Hence technically only one sperm is required for each ICSI. Various techniques have been described for sperm retrieval through testicles in azoospermic males. They include open surgery methods like open testicular biopsy (open TESE), MESA (Microscopic epididymal sperm aspiration), micro-TESE (Microscopic Testicular sperm extraction) and percutaneous methods like TESE (Testicular sperm extraction), PESA (Percutaneous epididymal sperm aspiration), TEFNA (Testicular fine needle aspiration). ${ }^{(10,11)}$

The sperm recovery rates ranges from $90 \%$ to $100 \%$ for obstructive azoospermia using any of standard surgical technique ${ }^{(12)}$, but the sperm recovery rates for non-obstructive azoospermia range from $30-63 \%$ for TESE, and $43-63 \%$ for Micro-TESE; the rate for TEFNA is approximately $47 \% .^{(17)}$ Out of all patients of azoospermia approximately $36 \%$ are due to obstructive azoospermia. ${ }^{(12)}$ Thus on an average overall surgical success rate for sperm retrieval ranges between $50 \%$ to $70 \%$. Thus in around $30 \%$ to $50 \%$ of azoospermic males even after aggressive open surgical exploration like MicroTESE we will not be able to retrieve sperms. If we could predict the outcome in such cases preoperatively we will be able to counsel the couple properly and will be able to give them other options like donor sperms use.

Assisted reproductive techniques (ART) for azoospermic couples involves procedure on both partners; ovum pickup in females and sperm retrieval in males. Thus in males with poor chances of recovery of sperms we may stage the ART in a way that if sperm recovery is successful then freeze or cryopreserve the sperms and then do ovulation induction and ovum pickup in female partner at later date. This will save unnecessary procedure and agony of female partner who is mostly a healthy partner. Economically also this could prove advantageous as couple could opt for donor intrauterine insemination in cases of failed sperm retrieval.

\section{Material and Methods}

This study was designed as a hospital based descriptive prospective study with the aim to find clinical, biochemical and intraoperative predictive factors for successful sperm retrieval and their predictive values amongst successful sperm retrieval. Also outcome of sperm retrieval procedures in azoospermic males in terms of successful retrievals by various surgical techniques was studied. Histopathological 
diagnosis, karyotyping, gene study, clinical pregnancy rates was assessed. This study aid in proper counseling of infertile couples.

\section{Inclusion Criteria}

1. Males of age above $21 \mathrm{yrs}$ and married

2. Male patients scheduled for surgical sperm retrieval for Artificial Fertilisation (ICSI)

3. Patients giving consent for use of their data in study.

\section{Exclusion Criteria}

1. All the patients who deny informed consent were excluded from the study.

\section{Methodology}

The institutional Review Board clearance was taken prior to the conduct of the study. It does not include any experimentation or deviation from normal surgical protocol. The patients were informed in detail about the study and written consent was obtained. Patient identity in any form was not revealed. None of the patient received any personal benefit by participating in the study. The records of all the patients treated in the Urology and department of IVF between February 2014 to February 2017 were reviewed.

80 patients who were azoospermic and who were admitted to hospital for surgical sperm retrieval as a part of Assisted Reproduction (ICSI) between were studied. After explaining the study to patients, informed consent was taken.

Various parameters of patients were recorded using data collection form.

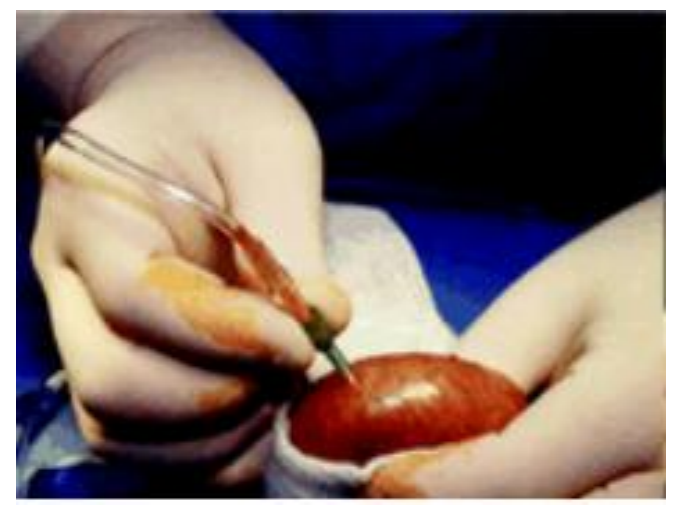

Fig 2. TESE-Testicular sperm extraction
Preoperative demographic data that was collected was patient age, height weight, BMI, years since marriage, semen analysis report for presence of fructose, serum hormone levels- FSH, LH, Testosterone and Prolactin and chromosomal analysis and gene analysis when available, history of orchitis, mumps or other clinically relevant history and examination was noted.

Right testicular volume and left testicular volume and mean of both testicle volume was noted. For testicular volume, if ultrasonography of scrotum was available then it was used. Where USG scrotum was not available prader orchiometer was used to estimate the size of testis. Condition of epididymis, presence of varicocele was noted. Patients with normal hormone levels, normal testis volume and dilated or full epididymis were classified as obstructive azoospermia and rest are classified as non-obstructive azoospermia patients. Those patients with dilated epididymis were offered PESA (percutaneous epididymal sperm aspiration) first, and if PESA failed to retrieve sperms then TESE (testicular sperm extraction) or micro TESE was offered during same sitting under local or sedation or general anaesthesia. Rest of the patients were offered TESE by needle aspiration biopsy technique using $18 \mathrm{G}$ scalp vein and applying negative suction. Four to six samples were collected from upper, middle and lower poles of testis and sent to IVF embryologist for screening. If sperms are found procedure is concluded and if sperms are not found other side TESE was done. If no sperms could be found by TESE then as per patients prior consent and counselling micro TESE was done.

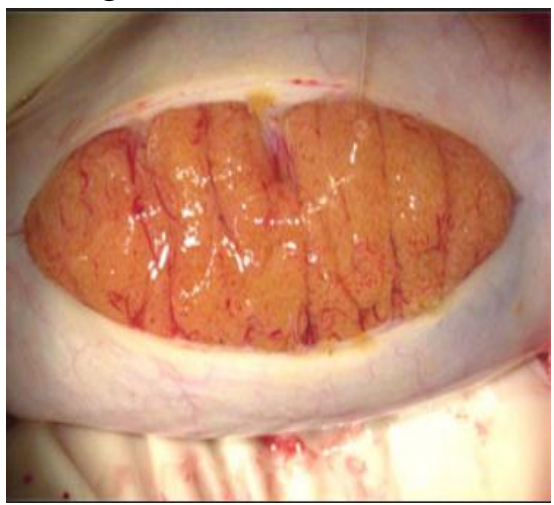

Fig 3. Micro TESE (Testis is bivalve) 


\section{JMSCR Vol||06||Issue||06||Page 128-139||June}

For micro TESE tunica is incised and testis parenchyma is bivalved along transverse axis. The seminiferous tubules are gently separated and examined under a Carl Zeiss operating microscope using $25 \mathrm{x}$ to $30 \mathrm{x}$ magnification. Healthy appearing or best available quality tubules were

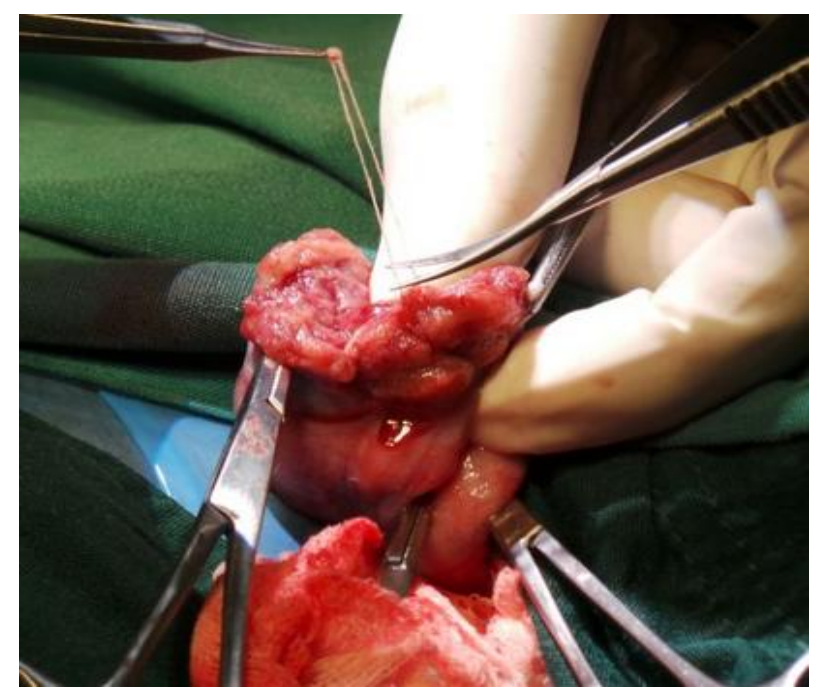

picked and sent for IVF embryologist screening. If no sperms are found procedure was repeated on opposite side. The quality of seminiferous tubules was noted and tubules were divided into 3 groups depending on most prominent appearance of tubule under operating microscope.

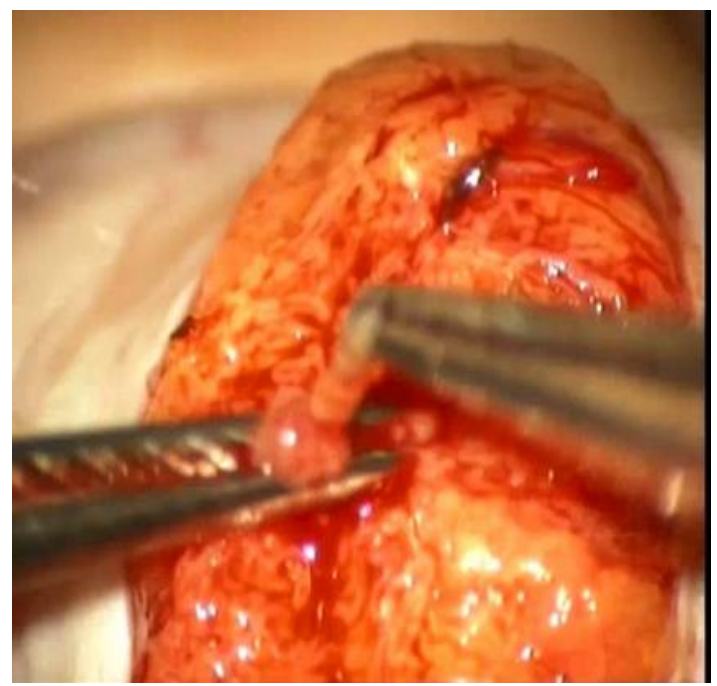

Fig 4. Semineferous tubules being picked up by micro-scissor

The groups are 1. Homogenous thin tubules 2 . Heterogeneous thin tubules with areas of atrophy and fibrosis 3. Thin gelatinous type where tubules are jelly like.
If no sperms could be found on initial screening or if the tubule quality is poor then some tissue was sent for histopathological examination.
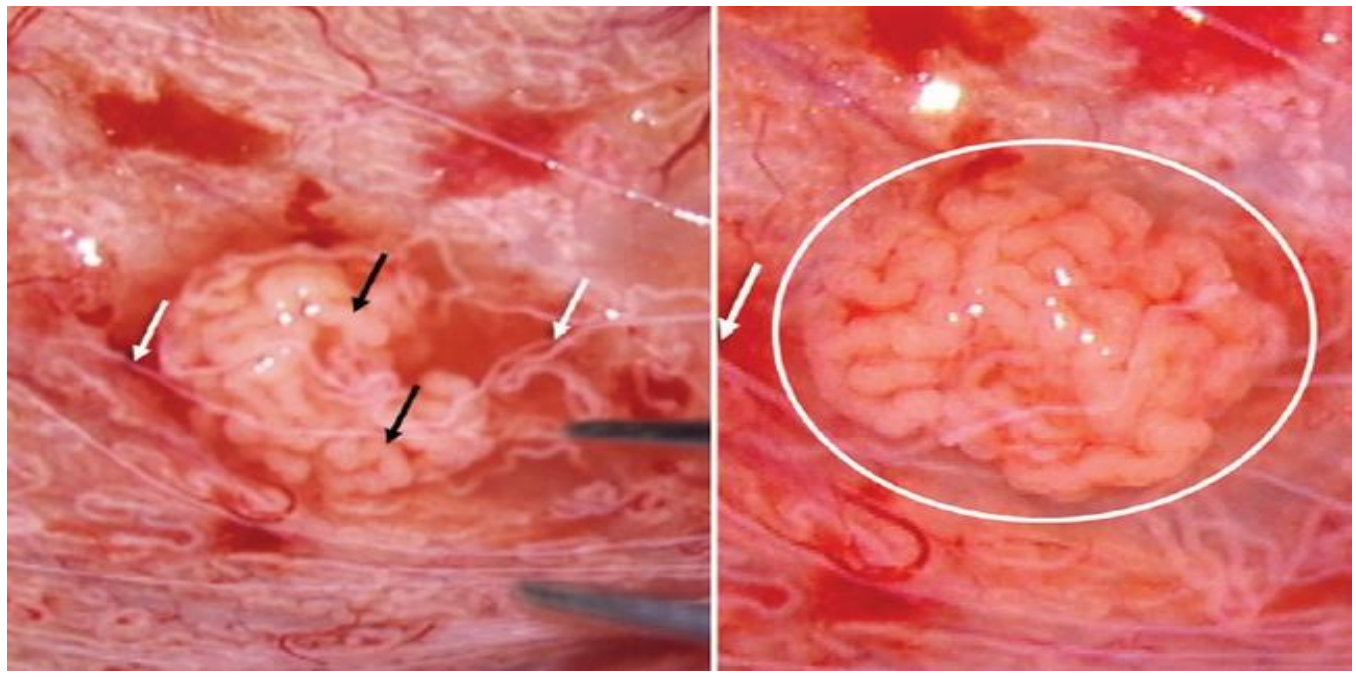

Fig 5. Microscopic appearance of seminiferous tubules (Thin homogeneous tubules)

Intraoperative findings, sperm retrieval status were noted in data collection form. Successful or unsuccessful pregnancies after successful sperm retrieval were followed up with the help of IVF doctors.

\section{Statistical Analysis}

Data collected in data collection form is tabulated under appropriate heading in MS Office Excel data Sheet. As per our study end point i.e usable sperm retrieval, the patients were divided into - 
successful or not successful group. For statistical analysis various variables were compared between these two groups. Mean of variables was calculated and used for analysis.

Qualitative data is represented in the form of frequency and percentage. Quantitative data is represented in the form of mean+/-sd, range.

Student $t$ test and Chi square test, fischer exact test were employed. SAS 9.2 and SPSS V15.0 (Statistical Package for Social Sciences, Version 15.0) were used to analyse the data. $P$ value $<0.05$ was considered as significant.

\section{Result}

In our study, out of 80 patients 9 patients had obstructive azoospermia (11.25\%) and 71 patients had non-obstructing azoospermia (88.75\%). Sperms were recovered in 45 patients and their mean age was found to be $35 \mathrm{yr} \& 1$ month. In 35 patients sperms could not be retrieved and their mean was 34 years $\& 5$ month. In non-obstructive azoospermia patients the mean BMI in patients with successful sperm retrieval was $27.53 \mathrm{~kg} / \mathrm{m}^{2}$ whereas in those with unsuccessful retrieval it was $28.98 \mathrm{~kg} / \mathrm{m}^{2}$. This difference in BMI is not statistically significant between two groups. In obstructive azoospermia group, all 9 patients had successful sperm retrieval and the mean BMI is found to be $26.43 \mathrm{~kg} / \mathrm{m}^{2}$. Year since marriage as an indirect indicator of infertility period was analysed. The mean infertility period in nonobstructive azoospermia patients with positive sperm retrieval was 6 year \&1 month and in negative sperm retrieval patients it was 4 year $\& 9$ month. The mean infertility period in obstructive azoospermia patients was 5 year 7 month. The mean FSH in non-obstructive azoospermia patients with positive sperm retrieval was 8.26 $\mathrm{mIU} / \mathrm{ml}$ and in negative sperm retrieval patients it was $14.61 \mathrm{mIU} / \mathrm{ml}$. On statistical analysis the FSH level between two groups shows significant difference. The mean FSH level in nonobstructive azoospermia patients was $3.47 \mathrm{mIU} / \mathrm{ml}$ and in all of them we retrieved sperms successfully. The mean LH level in non- obstructive azoospermia patients with positive sperm retrieval was found to be $8.85 \mathrm{mIU} / \mathrm{ml}$ and in negative sperm retrieval patients it was $9.04 \mathrm{mIU} / \mathrm{ml}$. On statistical analysis, LH level between two groups shows significant difference. In obstructive azoospermia patients mean LH is found to be $3.73 \mathrm{mIU} / \mathrm{ml}$. The mean testosterone level in non-obstructive azoospermia patients with positive sperm retrieval is found to be $436.81 \mathrm{ng} / \mathrm{dl}$ and in negative sperm retrieval patients it is $375.81 \mathrm{ng} / \mathrm{dl}$. On statistical analysis testosterone level between two groups do not shows significant difference. The mean testosterone level in obstructive azoospermia patients is found to be $560.8 \mathrm{ng} / \mathrm{dl}$. The mean prolactin level in nonobstructive azoospermia patients with positive sperm retrieval is found to be $6.29 \mathrm{ng} / \mathrm{ml}$ and in negative sperm retrieval patients it is $8.10 \mathrm{ng} / \mathrm{ml}$. On statistical analysis prolactin level between two groups shows significant difference. Mean prolactin level in patients with obstructive azoospermia is found to be $5.94 \mathrm{ng} / \mathrm{ml}$ which is within normal range.

Mean testis volume is an average single testis volume as calculated by dividing both testis volume by two. In non-obstructive azoospermia patients with positive sperm retrieval it is found to be $17.84 \mathrm{cc}$ and in negative sperm retrieval patients it is $12.32 \mathrm{cc}$. On statistical analysis the average testis volume between two groups shows significant difference. The mean testis volume in obstructive azoospermia patients is found to be $22.73 \mathrm{cc}$.

The sensitivity, specificity, positive predictive value, negative predictive value were calculated for serum FSH, LH, testosterone, prolactin and testicular volume. The cut off level for FSH was taken as $10 \mathrm{mIU} / \mathrm{ml}$, for $\mathrm{LH}$ as $10 \mathrm{mIU} / \mathrm{ml}$, for testosterone as $270 \mathrm{ng} / \mathrm{dl}$ and for prolactin as 14 $\mathrm{ng} / \mathrm{ml}$. The cut off for testis volume was taken as $10 \mathrm{cc}$. These cut off are based upon normal range of these factors in humans.

PESA surgery was done in 13 patients as they had dilated epididymis. In 9 patients sperms were retrieved. All these 9 patients had obstructive 
azoospermia. Out of 71 patients with nonobstructive azoospermia TESE was successful in retrieving sperms in 28 patients $(39.4 \%)$. Out of 43 unsuccessful TESE patients micro TESE was done in 34 patients only. In 9 patients micro TESE was not performed. Out of 34 micro TESE patients, sperms could be found in 8 patients (23.5\%). In cases of obstructive azoospermia ( $\mathrm{n}$ $=9$ ), sperm retrieval rate was $100 \%$ vis a vis of non-obstructive azoospermia $(\mathrm{n}=71)$, where sperm retrieval rate was $50.70 \%$.

As per microscopic appearance of seminiferous tubules, in homogenous tubule group out of 18 cases, only in 1 case sperms could be retrieved. In remaining 17 cases sperms could not be retrieved. In heterogenous thin atrophic tubule group out of 13 cases, in 6 cases sperms could be retrieved and in remaining 7 sperms could not be retrieved. In thin gelatinous tubule group out of 3 cases, in 1 case sperm is retrieved and in 2 cases sperm could not be retrieved.

Histopathological diagnosis was available for 33 patients. In 12 cases of severe hypospermatogenesis sperm could be found in 9 cases (75\%). In 10 cases of sertoli cell only syndrome only in $1(10 \%)$ case sperm could be found and in 11 cases of maturation arrest sperms were found in 4 cases (36.37). Chromosomal analysis and $\mathrm{Y}$ chromosome gene analysis was available in 18 cases. 15 patients had normal chromosomal study and no micro deletion on $\mathrm{Y}$ chromosome. In 2 patients 47XXY chromosome consistent with klinefelter's syndrome was found. In one patient microdeletion at $\mathrm{AZF}_{\mathrm{c}}$ gene on chromosome was found. Out of 80 patients including obstructive and non-obstructive azoospermia, sperm were found in 45 patients. Pregnancy data is available in 37 patients. Clinical pregnancy was achieved in 19 cases i.e $51.35 \%$ cases.

\section{Discussion}

In our study of 80 azoospermic males 9 (11.25\%) were diagnosed as obstructive azoospermia based on their normal hormone levels and normal size testis with fullness of epididymis. Remaining
$71(88.75 \%)$ were diagnosed as non-obstructive azoospermia patients. Obstructive azoospermia accounts for $35 \%$ to $40 \%$ cases of azoospermic males as per larger studies ${ }^{(6,11,13,14)}$. In our study, men with obstructive azoospermia planned for surgical retrieval are only $11.25 \%$. This may be because in many obstructive azoospermia cases vaso-epididymal anastomosis (VEA) surgery is offered to patients to get sperms in semen.

In our study the mean age of patient at the time of sperm retrieval procedure is $34 \mathrm{yr} 8$ month, youngest is 26yr 1 month old and oldest is 54 yr 3 months old. In patients with successful sperm retrieval mean age is $35 \mathrm{yr} 1$ month and in patients with unsuccessful sperm retrieval mean age is 34 yr 5 month. Similar age distribution is found in study by Ziaee et al. where mean age amongst successful and unsuccessful sperm retrieval patients ranges between $33 \mathrm{yr}$ to 36 yrs. ${ }^{(15)}$ The age difference is not statistically significant between successful and unsuccessful sperm retrieval patients.

In our study BMI of patients ranges from 19.5 $\mathrm{kg} / \mathrm{m}^{2}$ to $43.17 \mathrm{~kg} / \mathrm{m}^{2}$. Mean BMI among successful sperm retrieval patients is $27.5 \mathrm{~kg} / \mathrm{m}^{2}$ and among unsuccessful sperm retrieval patients is $28.9 \mathrm{~kg} / \mathrm{m}^{2}$. The difference in BMI is not statistically significant among successful and unsuccessful sperm retrieval patients. Study by Ramasamy $\mathrm{R}$ et al. also found similar results ${ }^{(16)}$ where sperm retrieval rates were similar in men with body mass index (BMI) $<25 \mathrm{~kg} / \mathrm{m}^{2}$ or $>30$ $\mathrm{kg} / \mathrm{m}^{2}$.

In our study infertility period in successful sperm retrieval patients was average 6 yr 1 month and in unsuccessful sperm retrieval patients was 4 yr 9 months. The difference in infertility period is not statistically significant between the two groups. But it may not represent true infertility period as it is calculated as years post marriage and not from the point when couple actually started trying to conceive due to lack of reliable sexual history of couple.

In our study we analysed level of hormones which influence spermatogenesis and have effect on 
hypothalamic-pituitary-gonadal axis. Hormones studied are FSH, LH, Testosterone and Prolactin. In our study the mean FSH level in 9 patients of obstructive azoospermia is found to be 3.47 $\mathrm{mIU} / \mathrm{ml}$ which is within normal range as expected. Other international studies also found normal FSH level in patients with obstructive azoospermia since the negative feedback mechanism for FSH is intact in obstructive azoospermia patients ${ }^{(7)}$.

In patients with non-obstructive azoospermia mean FSH level in patients with successful sperm retrieval is $8.2 \mathrm{mIU} / \mathrm{ml}$ which falls within normal FSH range (0.95 $\mathrm{mIU} / \mathrm{ml}$ to $11.95 \mathrm{mIU} / \mathrm{ml}$ ) (a7) whereas mean FSH level in patients with unsuccessful sperm retrieval is $14.61 \mathrm{mIU} / \mathrm{ml}$ which is higher than normal range. This difference in FSH level between two groups is statistically significant. Similar results are found in many international studies. ${ }^{(15,17,18,19)}$. The predictive power of serum FSH for successful sperm retrieval is also analysed. Tsujimura et al. have reported that preoperative serum FSH level in combination with other factors may help predict the success of micro-TESE. ${ }^{(20)}$ For serum FSH we found sensitivity to be $75 \%$, specificity to be $74.3 \%$, positive predictive value to be $75 \%$, negative predictive value to be $74.3 \%$ and diagnostic accuracy is found to be $74.65 \%$ in estimating chances of positive sperm retrieval. The cut off for FSH level was determined as $10 \mathrm{mIU} / \mathrm{ml}$. Ziaee $\mathrm{S} \mathrm{A}$ et al, in their similar study from Iran ${ }^{(15)}$ found predictive power of FSH for predicting successful retrieval much higher than our study i.e they found sensitivity to be $97 \%$, specificity to be $83.3 \%$, positive predictive value to be $88.2 \%$, negative predictive value to be $95.5 \%$. In their study the mean FSH in patients with successful sperm retrieval was $5.83 \mathrm{mIU} / \mathrm{ml}$, much lower than our study and in patients without successful sperm retrieval mean FSH level was $20.82 \mathrm{mIU} / \mathrm{ml}$, much higher than our study. In a detailed meta-analysis Yang $Q$ et al. showed heterogeneous predictive power of serum FSH as predictor of sperm retrieval and that region had an influence on the diagnostic value of FSH.
In our study the mean LH level in 9 patients of obstructive azoospermia is found to be 3.73 $\mathrm{mIU} / \mathrm{ml}$ which is within normal range (1.8 $\mathrm{mIU} / \mathrm{ml}$ to $12.07 \mathrm{mIU} / \mathrm{ml})$. Other studies also found normal $\mathrm{LH}$ level in patients with obstructive azoospermia.

In patients with non-obstructive azoospermia mean LH level in patients with successful sperm retrieval is $5.85 \mathrm{mIU} / \mathrm{ml}$ which falls within normal range whereas mean $\mathrm{LH}$ level in patients with unsuccessful sperm retrieval is $9.04 \mathrm{mIU} / \mathrm{ml}$ which is also in normal range. This difference in LH level between two groups is statistically significant. In study by Zhang et al. ${ }^{(21)}$ the mean level of LH is found to be higher than our study in both groups. In other studies the mean LH in both groups is similar to our study findings.

The predictive power of serum LH for successful sperm retrieval as found in our study are sensitivity of $83.3 \%$, specificity of $40 \%$, positive predictive value $58.8 \%$, negative predictive value to be $70 \%$ and diagnostic accuracy is found to be $61.97 \%$ in estimating chances of positive sperm retrieval.

Mean testosterone level in patients with obstructive azoospermia is found to be 560.8 $\mathrm{ng} / \mathrm{dl}$. It falls within normal range $(270 \mathrm{ng} / \mathrm{dl}$ to $1070 \mathrm{ng} / \mathrm{dl})$.

The mean testosterone level in non-obstructive azoospermia patients with successful sperm retrieval is $436.81 \mathrm{ng} / \mathrm{dl}$ and for patients with unsuccessful sperm retrieval is $375.81 \mathrm{ng} / \mathrm{dl}$. This difference of testosterone between successful and unsuccessful sperm retrieval patients is not signifycant statistically. A study by Zhang S et al. in 2015 shows similar testosterone levels in patients with successful and unsuccessful sperm retrieval. $^{(21)}$

Testosterone shows poor predictive power for predicting sperm retrieval. ${ }^{(15,21)}$ In our study for predicting successful sperm retrieval the sensitivity was $25 \%$, specificity was $62 \%$, positive predictive value was $44.90 \%$ and negative predictive value was $43.66 \%$. 
Serum prolactin levels were measured for azoospermic males. In patients with obstructive azoospermia the mean prolactin level is found to be $5.94 \mathrm{ng} / \mathrm{ml}$. The normal range of prolactin is 2 $\mathrm{ng} / \mathrm{ml}$ to $18 \mathrm{ng} / \mathrm{ml}$.

In patients with non-obstructive azoospermia who had successful sperm retrieval the mean serum prolactin level is found to be $6.29 \mathrm{ng} / \mathrm{ml}$ and in unsuccessful sperm retrieval patients it was 8.10 $\mathrm{ng} / \mathrm{ml}$. Tournaye $\mathrm{H}$ et al. found prolactin levels as poor predictors of successful sperm retrieval. ${ }^{(22)}$ In our study prolactin showed poor predictive values. The sensitivity was $94.4 \%$, the specificity was $5.7 \%$, positive predictive value was $50.70 \%$ and negative predictive value was $50 \%$.

The mean volume of testis in azoospermic males was analysed. Mean volume is calculated as addition of both testis volume divided by two. In obstructive azoospermia patients mean testis volume was $22.73 \mathrm{cc}$ whereas in patients with non-obstructive azoospermia in successful sperm retrieval group mean testis volume was $17.84 \mathrm{cc}$ and in unsuccessful retrieval group meam testis volume was $12.32 \mathrm{cc}$. This difference is statistically significant. Ziaee $\mathrm{S}$ A et al reported mean testis volume in successful sperm retrieval as $17.5 \mathrm{cc}$ similar to our study but in his study the mean testis volume in unsuccessful sperm retrieval patients was $5.68 \mathrm{cc}$ much less than our values. $\left.{ }^{(15}\right)$ Ziaee $\mathrm{S} A$ et al. and Tunc $\mathrm{L}$ et $\mathrm{al}^{(18)}$ report that testis volume correlate well with successful sperm retrieval. In our study as predictor of successful sperm retrieval the mean testis volume shows sensitivity of $84.4 \%$, specificity of $45.70 \%$, positive predictive value of $66.70 \%$ and negative predictive value of $69.60 \%$ and diagnostic accuracy of $67.50 \%$. Ziaee S A et al. in their study showed sensitivity of $88.9 \%$, specificity of $94 \%$, positive predictive value of $80 \%$ and negative predictive value of $69.60 \%$ and diagnostic accuracy of $96.90 \%$. Ziaee S A et al. concluded that testicular volume is an influential characteristic in successful sperm retrieval but topographical variations in testicular pathology independent of testicular volume can occur. Thus, testicular volume may not be a good predictive factor of successful sperm retrieval

In our study we performed PESA for 13 patients in whom the epididymis felt full or dilated on palpation. Out of 13 patients sperms were found in 9 patients (69.23\%). PESA is successful in all 9 cases of obstructive azoospermia. Lin Y M et al. found success rate for PESA to be $61 \%$ in their study published in $2000 .^{(23)}$

In our study TESE is done for patients with nonobstructive azoospermia. TESE alone succeeded in retrieving sperms in 28 patients (39.43\%). Out of 28 patients unilateral TESE succeeded in 22 patients and in 6 patient sperms were found after doing TESE on opposite side when sperms were not found on first side TESE. Hauser et al. in their article in 2006 reviewed TESE sperm recovery rates published in literature. ${ }^{(24)}$ They noted TESE sperm recovery rates (SRR) were $63 \%$ by Ezeh et al. $33 \%$ by Qublan et al. $40.8 \%$ by Aridogan et al. in his own study Hauser found TESE SRR of $62.1 \%$.

In our study 9 patients in whom sperms were not found by bilateral TESE micro TESE was not done. In remaining 34 micro TESE was done. Out of 34 patients in whom micro TESE was done, sperms were successfully retrieved in 8 patients i.e $11.26 \%$ of total patients or $23.5 \%$ of micro TESE patients. Dabaja AA et al. in their review ${ }^{(25)}$ on micro TESE published in 2013 noted significantly higher SRR(sperm retrieval rate) with microTESE $47 \%$ vs. $30 \%$ in conventional TESE.(26) Additional retrospective studies that compared conventional TESE with micro-TESE showed $16.7 \%$ SRR for TESE vs.44.6\% SRR with microTESE. Okada et al. 2002; Tsujimura et al. 2002 have reported a higher efficacy by micro TESE in yielding positive sperm recovery, even when multiple TESE is performed ${ }^{(27)}$ Our study show lower SRR for micro TESE $8 / 34$ i.e $23.5 \%$ as compared to other series.

In our study overall SRR in obstructive azoospermia is $100 \%$ for 9 patients of obstructive azoospermia out of cohort of 80 men. All positive sperm retrievals are by PESA. 
In our study overall SRR in non-obstructive azoospermia is $50.7 \%$ i.e 36 out of 71 patients of non-obstructive azoospermia. Bernie A, Ramasamy R et al. in 2013 mentioned that with use of micro-TESE, successful sperm retrieval has been reported in non-obstructive azoospermic men in up to $63 \%$ of patients and for obstructive azoospermia overall SRR is above 90\%. ${ }^{(28)}$ Various studies mention SRR in cases of nonobstructive azoospermia to be between $50 \%$ to $65 \%$ using all available surgical procedures.

Microscopic dissection TESE shows higher SRR because under $10 \mathrm{x}$ to $25 \mathrm{x}$ magnification of microscope we can see areas of focal spermatogenesis and pickup healthy appearing full seminiferous tubules. This focal spermatogenesis is the cause of unsuccessful conventional TESE. In our study we analysed the appearance of tubules we seen under microscope. The appearance is divided into 3 subtypes depending on prominent appearance. Tubules were grouped into homogenous appearing tubules, heterogeneous appearance of tubule with areas of thin atrophic tubules and thin gelatinous tubules where tubules are poorly formed and are jelly like. Homogenous tubules were present in 18 cases i.e $52.9 \%$ of total and in 17 cases sperm retrieval was unsuccessful. Heterogeneous tubules were seen in 13 cases i.e $38.23 \%$ of total and in 6 cases sperms were retrieved. Thin gelatinous tubules were found in 3 cases and in 1 case sperms were retrieved. The difference has been found statistically significant but this requires validation by analysing data in larger number of cases.

In literature not many articles mention dividing tubules as per their microscopic features and comparing sperm retrieval rates. But Ashraf MC in this study of micro TESE published in 2013 mentions clear cut microscopic identification of tubules into collapsed or full tubules and full tubules should be picked assuming better spermatogenesis. ${ }^{(29)}$

In our study, out of 71 patients of non-obstructive azoospermia in 33 patients tissue was sent for histopathological examination. 12 patients had hypospermatogenesis and sperms were retrieved in $9(75 \%)$ of them. 10 patients had sertoli cell only syndrome (SCO) and in only $1(10 \%)$ sperms could be retrieved. 11 patients had maturation arrest and in $4(36.36 \%)$ sperm could be retrieved. Glina $\mathrm{S}$ et al in their study published in 2005 found that $(\mathrm{d} 8)$ following surgical procedure for sperm retrieval, spermatozoa was found in $33 \%$ of the procedures in patients with Maturation arrest, $50 \%$ in patients with Hypospermatogenesis and in $40 \%$ of the procedures in patients with SCO.

In our study chromosomal analysis and AZF gene deletion studies were available in 18 patients. 15 patients had normal XY genotype, 2 patients had XXY Klinefelter's syndrome (KS) and one had $\mathrm{AZF}_{\mathrm{c}}$ deletion on $\mathrm{Y}$ chromosome. In klinefelter patients and in $\mathrm{AZF}_{\mathrm{c}}$ microdeletion patient sperms could not be retrieved. Sadeghi-Nejad H et al. in their review article published in $2007^{(31)}$ found that men with azoospermia and AZFc deletions may have retrievable sperm in their testes. However, they will transmit the deletions to their male offspring by intracytoplasmic sperm injection. Dabaja et al in his study in 2013 reported SRR in klinefelter syndrome patients to be between $60 \%$ to $65 \%{ }^{(25)}$

The pregnancy rates in our study are $51.35 \%$ similar to various studies. Miyaoka R et al. in their article published in 2013 mention pregnancy rates between $31 \%$ to $43 \%$ after using surgically retrieved sperms for ICSI. ${ }^{(12)}$ Dabaja et al. in his study published in 2013 found pregnancy rate of $48 \%$ by using retrieved sperms ${ }^{(25)}$.

\section{Limitations}

Infertility period was calculated as period since marriage and not as actual commencement of unprotected intercourse, as reliable sexual history was grossly lacking amongst couples. Since the duration of study and follow up period was only 3 years data for actual pregnancy rate is lacking in many cases. The study was further limited by histopathology report and chromosomal analysis/ gene analysis as it was not available for all patients. 


\section{Conclusion}

Age, BMI among successful and unsuccessful sperm retrieval groups are comparable and don't show any statistically significant difference. Whereas FSH, LH, Prolactin levels and mean testis volume show statistically significant difference between successful and unsuccessful sperm retrieval patients. High FSH, LH, prolactin and low testis volume are predictors of poor sperm retrieval rates but their predictive value is limited. Sperm retrieval for obstructive azoospermia gives excellent result with PESA. For non-obstructive azoospermia patients sperm retrieval can be achieved in around $50 \%$ of cases. Micro TESE increases overall sperm retrieval rates by around $12 \%$ when done after failed TESE. Microscopic seminiferous tubule appearance can be used to predict chance of sperm retrieval but requires validation by further studies and clinical pregnancy rates of around $50 \%$ can be achieved by using surgically retrieved sperms.

\section{References}

1. Zegers-Hochschild F, Nygren KG, Adamson GD, de Mouzon J, Lancaster P, Mansour R, Sullivan E. International Committee for Monitoring Assisted Reproductive Technologies. The ICMART glossary on ART terminology. Hum Reprod 2006;21:1968-70.

2. Brugh VM, Lipshultz LI. "Male factor infertility". Medical Clinics of North America 88 (2): 367-85.

3. Hirsh A. "Male subfertility" BMJ 327 (7416): 669-72.

4. Mascarenhas MN, Flaxman SR, Boerma T, Vanderpoel S, Stevens GA (2012) National, Regional, and Global Trends in Infertility Prevalence Since 1990: A Systematic Analysis of 277 Health Surveys. PLoS Med 9(12): 12243.

5. Jarow JP, Espeland MA, Lipshultz LI. Evaluation of the azoospermic patient. J Urol. 1989;142(1):62-5.
6. Schlegel P N. Causes of azoospermia and their management. Reprod. Fertil. Dev. 2004; 16:561-572.

7. Jarvi, K; Lo, K; Fischer, A; Grantmyre, J; Zini, A; Chow, V; Mak, V. "CUA Guideline: The workup of azoospermic males". Canadian Urological Association journal. 2010;4 (3): 163-7.

8. Silber SJ, Nagy ZP, Liu J, Godoy H, Devroey $\mathrm{P}$, Vansteirteghem AC. Conventional in-vitro fertilization versus intracytoplasmic sperm injection for patients requiring microsurgical sperm aspiration. Hum Reprod.1994;9 (9):1705-9.

9. Boulet SL, Mehta A, Kissin DM, Warner L, Kawwass JF, Jamieson DJ (2015). "Trends in use of and reproductive outcomes associated with intracytoplasmic sperm injection". JAMA. 2015;313 (3): 255-63.

10. Esteves SC, Miyaoka R, Agarwal A. Sperm retrieval techniques for assisted reproduction. Int Braz J Urol. 2011;37(5):570-83.

11. Pantke P, Diemer T ,Marconi M, Bergmann M, Steger K, Schuppe H, Weidner W. Testicular Sperm Retrieval in Azoospermic Men. Eur Urol 2008 (suppl 7) :703-714.

12. Miyaoka R, Esteves SC. Predictive factors for sperm retrieval and sperm injection outcomes in obstructive azoospermia: do etiology, retrieval techniques and gamete source play a role? Clinics (Sao Paulo)2013;68(Suppl 1):111-9.

13. Schwarzer JU, Fiedler K, v. Hertwig I, Kru“smann G, Wu“ rfel W, Schleyer M, Mu“ hlen B, Pickl U, Lo“ chner-Ernst D. Sperm retrieval procedures and intracytoplasmatic spermatozoa injection with epididymal and testicular sperms. Urol Int 2003;70:119-23.

14. Vernaeve V, Verheyen G, Goossens A, Steirteghem A Van, Devroey P, Tournaye H. How successful is repeat testicular sperm extraction in patients with azoospermia? Hum Reprod 2006;21:1551-4.

15. Ziaee SA, Ezzatnegad M, Nowroozi M, Jamshidian H, Abdi H, Hosseini Moghaddam 
SM: Prediction of successful sperm retrieval in patients with nonobstructive azoospermia. Urol J 2006, 3(2):92-96.

16. Ramasamy R, Bryson C, Reifsnyder JE, Neri Q, Palermo GD, Schlegel PN. Overweight men with nonobstructive azoospermia have worse pregnancy outcomes after microdissection testicular sperm extraction. Fertil Steril. 2013 Feb;99(2):372-6.

17. Glina S, Vieira M. Prognostic factors for sperm retrieval in non-obstructive azoospe-rmia. Clinics (Sao Paulo). 2013;68(Suppl 1):121-4.

18. Tunc L, Kirac M, Gurocak S, Yucel A, Kupeli B, Alkibay T, Bozkirli I: Can serum Inhibin B and FSH levels, testicular histology and volume predict the outcome of testicular sperm extraction in patients with non-obstructive azoospermia? Int Urol Nephrol 2006, 38(34):629-635.

19. Seo J, Ko W. Predictive factors of successful testicular sperm recovery in non-obstructive azoospermia patients. Int $\mathrm{J}$ Androl. 2001;24:306-10.

20. Tsujimura A, Matsumiya K, Miyagawa $\mathrm{Y}$. Prediction of successful outcome of microdissection testicular sperm extraction in men with idiopathic nonobstructive azoospermia. J Urol. 2004;172:1944-7.

21. Zhang S, An Y, Li J, Guo J, Zhou G, Li J, Xu Y. Relation between the testicular sperm assay and sex hormone level in patients with azoospermia induced by mumps. Int J Clin Exp Med 2015;8(11):21669-21673.

22. Tournaye H, Verheyen G, Nagy P, Ubaldi F, Goossens A, Silber S, et al. Are there any predictive factors for successful testicular sperm recovery in azoospermic patients? Hum Reprod. 1997;12:80.

23. Lin YM, Hsu CC, Kuo TC, Lin JS, Wang ST, Huang KE. Percutaneous epididymal sperm aspiration versus microsurgical epididymal sperm aspiration for irreparable obstructive azoospermia--experience with 100 cases. J Formos Med Assoc. 2000 Jun;99(6):459-65.
24. Hauser R, Yogev L, Paz G, Yavetz H, Azem F, Lessing JB, et al. Comparison of efficacy of two techniques for testicular sperm retrieval in nonobstructive azoospermia: Multifocal testicular sperm extraction versus multifocal testicular sperm aspiration. J Androl. 2006;27(1):28-33.

25. Dabaja A A and Schlegel P. Micro dissection testicular sperm extraction: an update. Asian J Androl. 2013; 15:35-39.

26. Amer M, Haggar SE, Moustafa T, Abd ElNaser T, Zohdy W. Testicular sperm extraction: impact of testicular histology on outcome, number of biopsies to be performed and optimal time for repetition. Hum Reprod 1999; 14:3030-4.

27. Okada H, Dobashi M, Yamazaki T, Hara I, Fujisawa M, Arakawa S, et al. Conventi-onal versus microdissection testicular sperm extraction for nonobstructive azoospermia. J Urology. 2002;168 (3):1063-7.

28. Tsujimura A. Microdissection testicular sperm extraction: prediction, outcome, and complications. Int J Urol. 2007;14(10):883-889.

29. Ashraf MC, Singh S, Raj D, Ramakrishnan S, Esteves SC. Micro-dissection testicular sperm extraction as an alternative for sperm acquisition in the most difficult cases of Azoospermia: Technique and preliminary results in India. J Hum Reprod Sci 2013;6:11123.

30. Glina S, Soares JB, Antunes N, Jr, Galuppo AG, Paz LB, Wonchockier R. Testicular histopathological diagnosis as a predictive factor for retrieving spermatozoa for ICSI in non-obstructive azoospermic patients. Int Braz J Urol. 2005;31(4):338-41.

31. Sadeghi-Nejad H, Farrokhi F. Genetics of azoospermia: current knowledge, clinical implications, and future directions. Part II: Y chromosome microdeletions. Urol J.2007;4(4):192-206. 